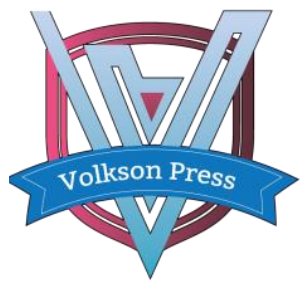

Contents List available at VOLKSON PRESS

New Materials and Intelligent Manufacturing (NMIM) DOI : http://doi.org/10.26480/icnmim.01.2018.73.75

Journal Homepage: https://topicsonchemeng.org.my/

ISBN: 978-1-948012-12-6

\title{
A FULL REFERENCE IMAGE QUALITY EVALUATION CORRESPOND WITH HUMAN VISUAL
}

\author{
Jin Hong-Jiao \\ College of Information and Control Engineering, Jilin Institute of Chemical Technology, Chengde Street, Jilin, China. \\ *Corresponding Author Email:
}

This is an open access article distributed under the Creative Commons Attribution License, which permits unrestricted use, distribution, and reproduction in any medium, provided the original work is properly cited

\section{ARTICLE DETAILS}

\section{Article History:}

Received 26 June 2018 Accepted 2 July 2018

Available online 1 August 2018

\section{ABSTRACT}

For the image edge blurring after inpainting, this paper propose a full-reference image quality assessment based on the human visual property. Using Weber-Fechner law, the modulation transfer function process and the B-spline wavelets constructing the direction tunable filter to simulate the human visual system. Establishing a visual evaluation model. The experiment derives the evaluation method has a high correlation with subjective evaluation.

\section{KEYWORDS}

Image inpainting quality, Ful-reference, Human Visual System (HVS)

\section{INTRODUCTION}

With the image restoration applying to many aspects, the quality of the image inpainted has been paid more attention. The quality of the image inpainted has the extent to accords with the needs of human visual; it is one of the important indicators to measure inpainting technology. Image quality evaluation has the subjective and objective method. The objective evaluation studied in this paper uses the algorithm to simulate the human eye subjective perception of image quality, there is three categories method depends on the reference image exists: the full reference, half reference and no reference [1].

Many evaluations are proposed to the simulation the HVS, Ding presents wavelet transform to simulate the characteristics of multi channel of HVS, the coefficient are weighted of the sub-band of different spatial frequency after transform, the weighted value is used to evaluate image quality [2]. Wang propose the structural similarity (SSIM). The structure information of the image is collected when observing an image, evaluating the similarity of two images in structure [3]. Sheikh propose VIF using natural scene statistics method to estimate the retention degree of information from the distortion image relative to the reference image, and evaluating the distortion degree by the fidelity [4]. The FSIM proposed by Zhang et al. uses the low-level feature to estimate the image quality before and after the distortion [5]. Another researchers proposes the gradient similarity based on gradient information in the image. These methods are considered to be effective [6]. This paper adopts the Weber-Fechner law, the modulation transfer function and the B-spline wavelet to structure the Human Visual System. Experimental result shows that this method is consistent with subjective perception.

\subsection{HVS property}

HVS (Human Visual System) model established simulates the characteristics of the human eyes in the process of evaluating image, that is the nonlinear damping in visual, two-dimensional visual sensitivity band-pass and visual multi-channel and masking effect.

\subsubsection{Visual nonlinearity}

Weber's law describes the visual nonlinearity. The image is divided into two parts when observed with its brightness is $\mathrm{L}$, and one part increases, $L+\nabla L$ is just the human eye can distinguish the difference, then $L$ increases or reduces in a certain range, the $\nabla L / L$ will not vary with $L$. Setting the visual brightness as $\mathrm{B}$, if it meet:

$$
\Delta B=\alpha \Delta L / L \quad \text { (1) Where a 、 } \beta \text { is the constant. }
$$

\subsubsection{D Visual sensitivity band-pass}

The visual contrast sensitivity is described by the modulation transfer function. The band-pass filter can describe the human visual transmission better through the study of human visual property.

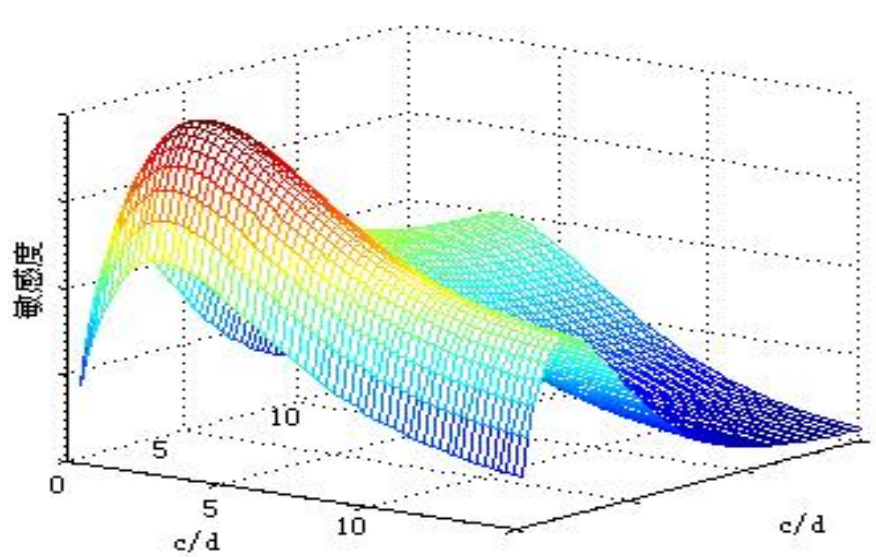

Figure 1: Modulation Transfer Function

Figure 1 indicates that the human eye is most sensitive at $2 \sim 5 c / d$.

\subsubsection{Visual multi-channel and masking effect}

Visual masking refers to the phenomenon that when the human eye observes an image, the presence of one region causes the human eye to ignore the effect of another region, and the visibility of the area is neglected.

2.FULL REFERENCE IMAGE QUALITY EVALUATION BASED ON HVS MODEL 


\subsection{HVS Evaluation Model Established}

The VMSE (Visual mean square error, VMSE) is defined according to the definition of objective quality evaluation formula. Two norm measure distortion measurement is adopted:

$$
\begin{gathered}
d(x 1 \bullet x 2)=\|x 1-x 2\|_{2}=\sum(x 1-x 2)^{2} \\
V M S E=\frac{E\left\{V\left(I(i, j)-I^{\prime}(i, j)\right) \|^{2}\right\}}{E\left(\|I(i, j)\|^{2}\right)}
\end{gathered}
$$

where, $V$ is the visual characteristic function; $I(I, j)$ is the original image; $I^{\prime}(I, j)$ is the image inpainted. That is:

$$
V M S E=\sum\left\{V\left[I(i, j)-I^{\prime}(i, j)\right]\right\}^{2} / \sum I(i, j)^{2}
$$

$\mathrm{V}$ is the meaning of the human eye visual impacting on image quality assessment, this paper transforms visual characteristic into three cascade relationship function:

$$
V=F\left(\Gamma_{1}\left(\sigma_{e}^{2}\right), \Gamma_{2}\left(\sigma_{e}^{2}\right), \Gamma_{3}\left(\sigma_{e}^{2}\right)\right)
$$

Where, $\Gamma 1(\sigma \mathrm{e} 2)$ is the amplitude nonlinear; $\Gamma 2(\sigma \mathrm{e} 2)$ is the twodimensional visual sensitivity function; $\Gamma 3(\sigma \mathrm{e} 2)$ is the visual detection function, $\sigma \mathrm{e} 2=\| \mathrm{I}(\mathrm{I}, \mathrm{j})-\mathrm{I}$ ' (i, j) $\|$ and $\sigma \mathrm{e} 2$ is the error gray value. The method for construction of evaluation model as follows:

(1) Weber-Fechner law is adopted to simulate the non-linear characteristics:

$\varepsilon_{w}=\Gamma_{1}\left(\sigma_{e}^{2}\right)=k\left[I(i, j)-I^{\prime}(i, j)\right]^{1 / 2.2}$

$\mathrm{K}$ is the constant.

(2) The band-pass characteristic of $2 \mathrm{~d}$ visual sensitivity is described by the modulation demodulation function:

$$
\begin{aligned}
& \varepsilon_{m}=\Gamma_{2}\left(\sigma_{e}^{2}\right)=\varepsilon_{w} * S(w) \\
& S(w)=1.5 e^{-\sigma^{2} w^{2} / 2}-e^{-2 \sigma^{2} w^{2}}
\end{aligned}
$$

Where the constant $\sigma=2$, the angular frequency $\omega=(2 \pi f / 60)$.

Calculating the spatial frequency value of each pixel of the image after inpainting and defining its horizontal spatial frequency $u$ and vertical spatial frequency v:

$$
\begin{gathered}
u=\sqrt{\frac{1}{M N} \sum_{m}^{M} \sum_{n=2}^{N}\left[I^{\prime}(i, j)-I^{\prime}(i, j-1)\right]^{2}} \\
v=\sqrt{\frac{1}{M N} \sum_{m=2}^{M} \sum_{n=1}^{N}\left[I^{\prime}(i, j)-I^{\prime}(i, j-1)\right]^{2}}
\end{gathered}
$$

Where $\mathrm{M}$ and $\mathrm{N}$ is the height and width of the image respectively, the spatial frequency is $f=\sqrt{u^{2}+v^{2}}$.

(3) B-spline wavelet to structure filter group. Introduce a theorem:

$$
\sum_{k=0}^{n-1} \cos ^{2 m}\left(\theta-\frac{k}{n} \pi\right)=\frac{(2 m-1) ! !}{(2 m) ! !} \quad, 1 \leq m \leq n-1,2 \leq n
$$

Theorem 1

The multi-scale filter group is established in polar coordinates. The angular frequency filter is:

$$
H_{k}(\theta)=j \cos ^{m}\left(\theta-\frac{k}{n} \pi\right) \quad 1 \leq m \leq n-1,2 \leq n, 0 \leq k \leq n-1
$$

Where $n$ is the number of directions selected, generally $n=4$. Radial low pass filter $\mathrm{H}(\mathrm{r})$, decomposition filter, and the recovery filter:

$$
\begin{gathered}
H(r)=(\cos (r / 2))^{n+1}, G(r)=-16 \sin ^{2}(r / 2), \\
G_{k}(r)=G(r) \bullet H_{k}(\theta)=-\frac{1}{16} \sum_{j=0}^{n}(\cos (r / 2)) \\
\tilde{G}_{k}(r)=\tilde{G}(r) \bullet \bar{H}_{k}(\theta)
\end{gathered}
$$

$\bar{H}_{k}(\theta)$ is the conjugate of $H_{k}(\theta)$. According to theorem 1 :

$$
H^{2}(r)+\sum_{k=0}^{n-1} G_{k} \tilde{G}_{k}=1
$$

Theorem 1 guarantees that the wavelet can be decomposed in any direction. Divide by $0^{\circ}, 45^{\circ}, 90^{\circ}, 135^{\circ}$ respectively:

$$
\begin{gathered}
S_{2^{j}} \varepsilon_{m}=S_{2^{j-1}} \varepsilon_{m} * h \\
W^{n}{ }_{2^{j}} \varepsilon_{m}=S_{2^{j-1}} \varepsilon_{m} * g_{n}
\end{gathered}
$$

The reduction formula is:

$$
S_{2^{j-1}} \varepsilon_{m}=S_{2^{j}} * \bar{h}+\sum_{n=0}^{3} W^{n}{ }^{j} \varepsilon_{m} * \bar{g}_{n}
$$

where $\mathrm{n}$ is the wavelet order, $2^{\mathrm{j}}$ is the wavelet scale, $\mathrm{h}$ and $\mathrm{h}$ is the time domain response of the decomposition filter $g_{n}$ and $\bar{g}_{n}$ is the time domain response of the recovery filter. $W^{n}{ }_{2^{j}} \mathcal{E}_{m}$ is n detail signals on the scale $2^{\mathrm{j}}$ for the image error signal $\varepsilon_{m}$. The error signal is decomposed by decomposing filter to obtain the error signal of two scales and four directions.

The error signal $\varepsilon_{m}$ is decomposed by decomposing filter to obtain the error signal $\varepsilon_{i}$ in two scale and 4 direction, $\mathrm{i}=1,2, \ldots, 2 \times 4+1$. These errors are cumulative to $V_{\varepsilon}$. The error $V_{\varepsilon}$ in the visual is obtained through the transformation of the physical error, the formula:

$$
V_{\varepsilon}=\Gamma_{3}\left(\sigma_{e}^{2}\right)=\sum_{i=1}^{9} \varepsilon_{i}=\sum_{n=0}^{3} \sum_{j=0}^{1} S_{2^{j-1}} \varepsilon_{m}
$$

Where $i=n * j+1, n=0 \ldots .3$ is four directions, $j=0,1$ is two scales.

\subsection{Evaluation Method}

The evaluation model is shown in figure 2. Original image I ( $\mathrm{i}, \mathrm{j})$, Inpainting image I'(i, j); Error segmentation SPE; Covariance COV; Multiple Regression Analysis (MRA):

Step1: obtaining error signaløe2 from I(i,j) and I'(i,j);

Step2: obtaining visual error signal Vefrom $\sigma \mathrm{e} 2$ calculated by visual evaluation model;

Step3: $V \varepsilon$ is divided into edge error $\left(E_{1}\right)$ and flat area error $\left(E_{2}\right)$;

Step4: the weight of edge error and flat region error is obtained through regression analysis, the formula of the visual mean square error:

VMSE $=5.254-0.853 \mathrm{E} 1-1.032 \mathrm{E} 2$

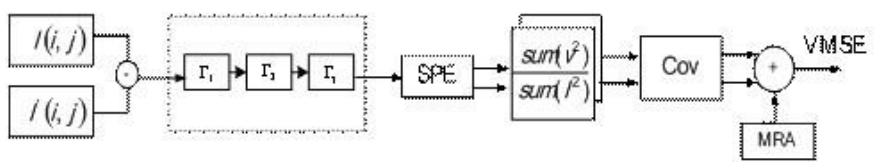

Figure 2: VMSE Evaluation Model

\subsection{Experimental results and analysis}

The test images are selected from the images inpainted in my lab. The subjective method selects the CCIR 500 recommendation of the five-level quality evaluation standard - MOS (Mean Opinion Score: MOS), three groups image are $256 \times 256,512 \times 512$ and $171 \times 512$ in size. The test images are shown in figures 3,4 and 5 . The results are shown in Table 1 and 2:
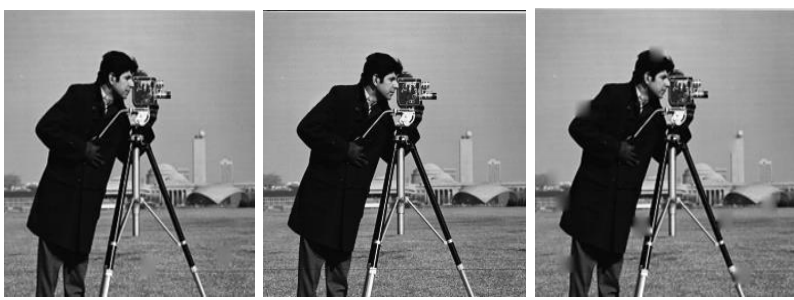
(a)

(b)

(c)

Figure 3: Inpainting Image

(a) Original image (b) Markov random field (MRF) human-computer interaction (c) MRF.

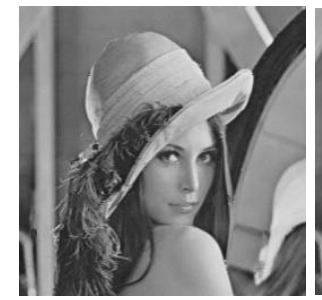

(a)

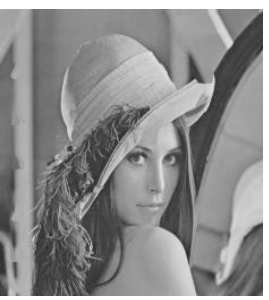

(b)

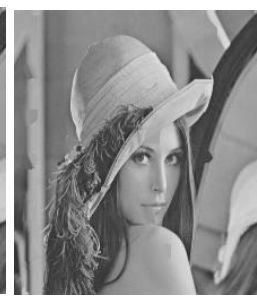

(c)
Figure 4: Inpainting Image

(a)Original image (b) Gauss MRF eight direction synthesis (c) Gauss MRF single direction

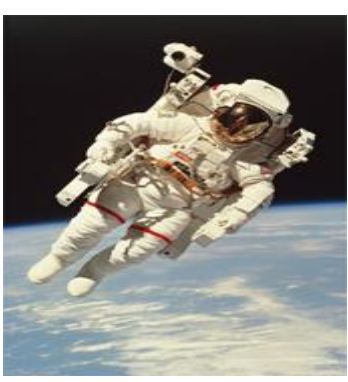

(a)

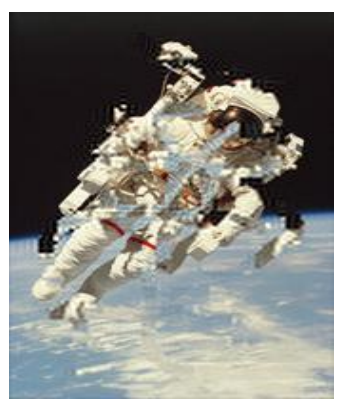

(c)

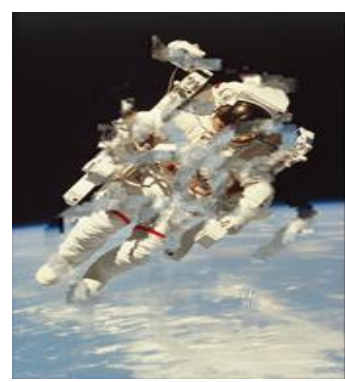

(b)

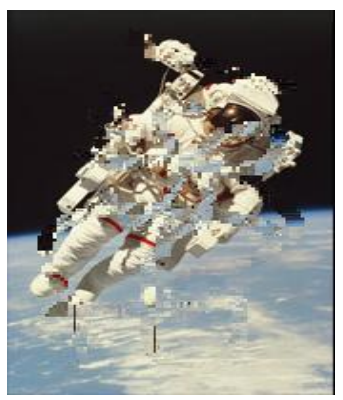

(d)
Figure 5: Inpainting Image

(a) Original image (b) Transform of double tree complex wavelet (c) Transform of Contour wave wavelet (d) Transform of discrete wavelet

Table 1: Objective Evaluation

\begin{tabular}{lll}
\hline Score & $P S N R$ & $V M S E$ \\
\hline Fig 2.6(b) & 61.4502 & 4.633 \\
Fig 2.6(c) & 61.4420 & 2.976 \\
Fig 2.7(b) & 70.4142 & 3.518 \\
Fig 2.7(c) & 70.4094 & 3.568 \\
Fig 2.8(b) & 18.6196 & 3.695 \\
Fig 2.8(c) & 18.6176 & 3.289 \\
Fig 2.8(d) & 18.0453 & 3.112 \\
\hline
\end{tabular}

Table 2: Subjective Evaluation

\begin{tabular}{lllllll}
\hline Score & \multicolumn{7}{c}{ The number of observers determine that } \\
the image is i score & \multicolumn{2}{l}{ Score } \\
Img & 5 & 4 & 3 & 2 & 1 & $\bar{C}$ \\
\hline Fig 2.6(b) & 3 & 10 & 2 & 0 & 0 & 4.067 \\
Fig 2.6(c) & 0 & 2 & 6 & 7 & 0 & 2.000 \\
Fig 2.7(b) & 0 & 1 & 7 & 6 & 1 & 2.533 \\
Fig 2.7(c) & 0 & 0 & 6 & 6 & 3 & 2.200 \\
Fig 2.8(b) & 0 & 2 & 6 & 6 & 1 & 2.600 \\
Fig 2.8(c) & 0 & 2 & 5 & 6 & 2 & 2.467 \\
Fig 2.8(d) & 0 & 0 & 4 & 10 & 1 & 2.200 \\
\hline
\end{tabular}

The experimental results of the objective evaluation method PSNR and the visual perception evaluation method VMSE in this paper are shown in table 1. The related degree of VMSE and MOS to describe correlation of this method with the human eye vision, the correlation is measured by the Pearson correlation coefficient $\mathrm{R}$ :

$$
R=\frac{\sum_{i=1}^{N}\left(X_{i}-\bar{X}\right) *\left(Y_{i}-\bar{Y}\right)}{\sqrt{\sum_{i=1}^{N}\left(X_{i}-\bar{X}\right)^{2} * \sum_{i=1}^{N}\left(Y_{i}-\bar{Y}\right)^{2}}}
$$

Where $\bar{X}=\sum_{i=1}^{N} \frac{X_{i}}{N}, \bar{Y}=\sum_{i=1}^{N} \frac{Y_{i}}{N}$ is the value of the sample $\mathrm{X}$ and $\mathrm{Y}$ respectively, $\mathrm{X}$ is the value of MOS , $\mathrm{Y}$ is the value of VMSE, $\mathrm{N}$ is the total of image samples, here $\mathrm{N}=7$. The correlation coefficient of calculation and subjective evaluation is 0.947 , which shows that this method is consistent high with human vision.

\section{CONCLUSION}

The evaluation method in this paper and the objective evaluation method are tested respectively, and the advantages and disadvantages of the two evaluation methods are analyzed according to the experimental results. The experiment proves that the evaluation method in this paper can consider the human eye vision better, and the evaluation result is better than the objective, which has a higher degree of correlation with the subjective method.

\section{REFERENCE}

[1] Sheikh, H.R., Sabir, M.F., Bovik, A.C. 2006. A statistical evaluation of recent full reference image quality assessment algorithms [J]. IEEE Transactions on Image Processing, 15(11).

[2] Xuxing, D. 2004. A Criterion of Image Quality Assessment Based on Property of HVS. Journal of Image and Graphics, 9 (2), 190-194.

[3] Wang, Z., Bovik, A.C., Sheikh, H.R. 2004. Image quality assessment: from error visibility to structural similarity[]. IEEE Transactions on Image Processing, 13.

[4] Sheikh, H.R., Bovik, A.C. 2006. Image information and visual quality [J]. IEEE Transactions on Image Processing, 2006, 15 (2).

[5] Zhang, L., Zhang, L., Mou, X.Q. 2011. FSIM: A feature similarity index for image quality assessment [J]. IEEE Transactions on Image Processing, 20 (8).

[6] Liu, A., Lin, W., Narwaria, M. 2012. Image quality assessment based on gradient similarity [J]. IEEE Transactions on Image processing , 21 (4). 